Note

\title{
Development of a Predictive Program for Vibrio parahaemolyticus Growth under Various Environmental Conditions
}

\author{
HIROSHI FUJIKAWA ${ }^{1 *}$, BON KIMURA ${ }^{2}$, AND TATEO FUJII ${ }^{2}$ \\ 1 Department of Veterinary Medicine, Tokyo University of Agriculture and Technology, \\ 3-5-8 Saiwai-cho, Fuchu, Tokyo 183-8509, Japan \\ ${ }^{2}$ Department of Food Science and Technology, Tokyo University of Marine Science and \\ Technology, 4-5-7 Konan, Minato, Tokyo 108-8477, Japan
}

Received 3 February, 2009/Accepted 24 April, 2009

\begin{abstract}
In this study, we developed a predictive program for Vibrio parahaemolyticus growth under various environmental conditions. Raw growth data was obtained with a $V$. parahaemolyticus 03:K6 strain cultured at a variety of broth temperatures, $\mathrm{pH}$, and salt concentrations. Data were analyzed with our logistic model and the parameter values of the model were analyzed with polynomial equations. A prediction program consisting of the growth model and the polynomial equations was then developed. After the range of the growth environments was modified, the program successfully predicted the growth for all environments tested. The program could be a useful tool to ensure the bacteriological safety of seafood.
\end{abstract}

Key words : Computer program/Predictive microbiology/Vibrio parahaemolyticus/Logistic model/ Polynomial model.

Nowadays, consumers tend to refrain from buying food products with food additives from the viewpoint of food safety. It is becoming considerably important to produce and distribute food products under hygienically good environmental conditions to decrease the possibility of microbial food poisoning outbreaks. Among environmental factors, temperature, hydrogen ion concentration $(\mathrm{pH})$, and water activity are thought to be the most influential factors.

On the other hand, predictive microbiology models can be a tool to reduce microbial food poisoning outbreaks. Growth models can predict microbial growth under various environmental conditions. The modified Gompertz model, Baranyi model, and a three-phase model are growth models that are well-known worldwide (Gibson et al., 1987; Baranyi et al., 1995; Buchanan et al., 1997). We also developed a growth model that was an extended model of the logistic model (Fujikawa et al., 2003 and 2004). The model

*Corresponding author. e-mail address: fujik@cc.tuat.ac.jp. successfully predicted bacterial growth at a variety of temperatures, very similar to the Baranyi model (Fujikawa and Morozumi, 2005 and 2007).

Furthermore, expert computer programs have been developed for use in the food industry. When users input environmental data such as temperature, $\mathrm{pH}$, and water activity into the program, it instantly predicts microbial growth and/or death. Pathogen Modeling Program is one of the examples of these programs (http://pmp.arserrc.gov/PMPHome.aspx). Combase is also a database program for predictive microbiology (http://wyndmoor.arserrc.gov/combase/).

However, no predictive programs for Vibrio parahaemolyticus growth under various environmental conditions have been developed so far. $V$. parahaemolyticus, a contaminant of seafood, is one of the major food poisoning pathogens, particularly in the Far East. The pathogen was first identified in Japan, where people often eat raw fish (Fujino et al., 1950). Recently, V. parahaemolyticus O3:K6 strains have been linked to epidemics of gastroenteritis worldwide (Okuda et al., 1997; Nair et al., 2007). The 
U.S. Food and Drug Administration and Center for Food Safety and Applied Nutrition reported a risk assessment on V. parahaemolyticus in seafood in 2001 (http://vm.cfsan.fda.gov/ dms/vprisk.html).

Thus, in this study, we developed a predictive program for $V$. parahaemolyticus growth. The program was built on our growth model using the experimental data of an 03:K6 strain (Kimura et al., 2008).

A new logistic model which we recently developed was used to develop the growth program (Fujikawa et al., 2003 and 2004; Fujikawa and Morozumi, 2005 and 2007). The model is shown in equation (1).

$$
\frac{d N}{d t}=r N\left\{1-\left(\frac{N}{N_{\max }}\right)^{m}\right\}\left\{1-\left(\frac{N_{\min }}{N}\right)^{n}\right\}
$$

where $N$ is the population of the organism $(\mathrm{CFU} / \mathrm{ml})$ at time $t(h), r$ is the rate constant of growth $(1 / \mathrm{h})$, $N_{\max }$ is the maximum population $(\mathrm{CFU} / \mathrm{ml})$, and $N_{\min }$ is the initial population $(\mathrm{CFU} / \mathrm{ml}) . m$ and $n$ are parameters related to the curvature of the deceleration phase and the period of the lag phase, respectively.

An 03:K6 strain, V03-80 was used as a test strain for this study (Kimura et al., 2008). The strain was selected among a total of $34 \mathrm{~V}$. parahaemolyticus strains studied (Kimura et al., 2008), because it is one of the strains that grew well at high salt concentrations (data not shown). Growth data of the strain were from a recent study done with the financial support of the Japan Food Industry Center (http://www. shokusan.or.jp/haccp/news/index_18.html).Namely, the organism was grown in Trypticase Soy broth. Salt was added to designated concentrations into the broth. $\mathrm{pH}$ values of the broth were adjusted with hydrogen chloride or sodium hydroxide. After inoculation of the organism, the broth was incubated at a given temperature with shaking. Samples were taken at intervals and colony counts were measured with Trypticase Soy agar plates containing 1.8\% salt.

The growth conditions were as follows. Temperatures were $15,20,25$, and $30^{\circ} \mathrm{C}$. The salt concentrations were $0.5,4.5,6.5$, and $8.9 \%$, which corresponded to Aw 1.0, 0.99, 097, and 0.955, respectively. $\mathrm{pH}$ values were $5.5,6.0,7.0$, and 8.0. All combinations of these conditions were tested for growth.

Bacterial growth data were analyzed with a fitting program for the growth model (Fujikawa and Kano, 2009). The program was developed on a spreadsheet calculation program, Microsoft Excel ${ }^{\circledR} 2003$. When we input growth data into the analysis program, it gave the best-fitted curve on a graph with the estimated values of parameters $r, m$, and $\mathrm{n}$. Data showing no growth at certain environmental factors could not be analyzed with the model.

Values of parameters $r, m$, and $n$ of the growth equation for data sets were analyzed with a polynomial model. An example for $r$ with a 3-order polynomial equation is shown as follows:

$$
\begin{aligned}
r= & a_{1} T^{3}+a_{2} S^{3}+a_{3} P^{3}+a_{4} T^{2} S+a_{5} S^{2} P+a_{6} P^{2} T \\
& +a_{7} T^{2} P+a_{8} S^{2} T+a_{9} P^{2} S+a_{10} S P+a_{11} T^{2}+a_{12} S^{2} \\
& +a_{13} P^{2}+a_{14} T S+a_{15} S P+a_{16} P T+a_{17} T+a_{18} S \\
& +a_{19} P+a_{20}
\end{aligned}
$$

where $T$ is temperature $\left({ }^{\circ} \mathrm{C}\right), S$ is the salt concentration of the broth (\%), and $P$ is $\mathrm{pH}$ of the broth. $a_{1}$, $a_{2}, \cdots, a_{20}$ are the parameters of the polynomial equation. The optimal values for the parameters $a_{1}$ to $a_{20}$ were estimated by the least square method between the observed and estimated values of $r$ with the Solver in Microsoft Excel $2003^{\circledR}$. The same procedures were done for $m$ and $n$.

When the growth data were analyzed with the new logistic model, those were successfully fitted with the model, as shown in Fig. 1. In some of the growth curves, the population gradually decreased after it reached the maximum. For those curves, the data up to the maximum population were analyzed with the fitting program.

Parameter values of the growth model under various environmental conditions were analyzed with 2-, 3 -, and 4-order polynomial equations. For $r$, as the number of order increased, the mean square error (MSE) between the observed and estimated values decreased; the MSE values for the 2-, 3-, and 4-order equations were $0.0522,0.0132$, and 0.0062 , respectively. However, the 4-order equation gave extraordinary estimates for some of the environments. Thus, the 3-order equation (Eq. 2) was applied to r. For $m$, since the MSE value was not small for the optimal values of parameters $a_{1}$ to $a_{20}$ (data not shown) and the value of $m$ itself did not considerably affect the shape of a growth curve, the value of $m$ was fixed to one. The MSE values for $n$ with the 2-, 3-, and 4-order equations were 17.2, 12.3, and 21.9, respectively. Thus, the 3-order equation (Eq. 2) was also applied to $n$.

With the parameter values of the growth model that were estimated by the above polynomial equations, growth curves under various environments were simulated. Most of those predicted curves were similar to the observed curves (Fig. 2A), but some of the curves were considerably different from the observed ones (Fig. 2B). Such poor prediction was due to the extraordinary values of $r$ and $n$ estimated with the 


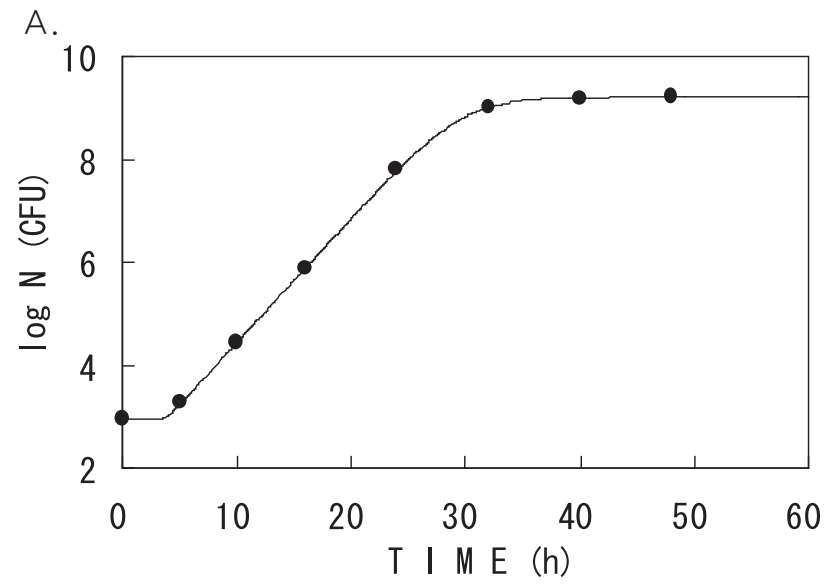

B.

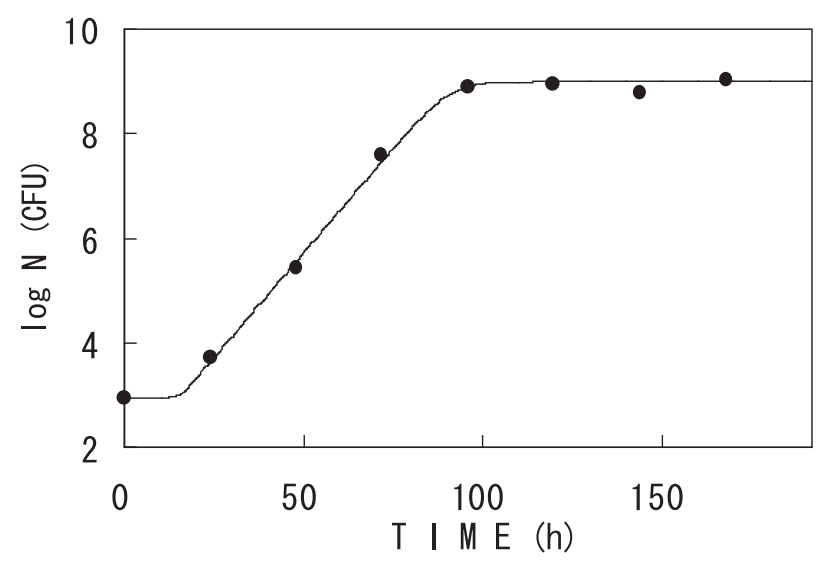

FIG. 1. Examples of the fitting performance of the growth analyzing program. Growth conditions are A. $20^{\circ} \mathrm{C}, \mathrm{pH} 7.0$, and $6.5 \%$ salt and $\mathrm{B} .20^{\circ} \mathrm{C}, \mathrm{pH} 7.0$, and $8.9 \%$ salt. Closed circles are experimental results. Curves are described with the new logistic model.

polynomial equations for those environmental conditions. Those conditions included the salt concentration of $8.9 \%$ or $\mathrm{pH} 5.5$.

For better prediction for $r$ and $n$ under every environmental condition, we reduced the region of prediction. That is, the new environmental range of conditions subject to prediction was reduced to the ranges of $0.5-6.5 \%$ salt and $\mathrm{pH} 6-8$. (The temperature range was not changed.) In this area, parameters $r$ and $n$ were analyzed with the polynomial equation again. As a result, we obtained better estimates for the parameters. The MSE values $r$ and $n$ with a 3order equation were 0.011 and 11.5 , respectively, which were both smaller than the former ones described above.

We further improved the prediction of bacterial population by introducing factors for parameters $r$ and $n$ obtained with the 3-order equations. That is, the factor for each parameter was estimated by

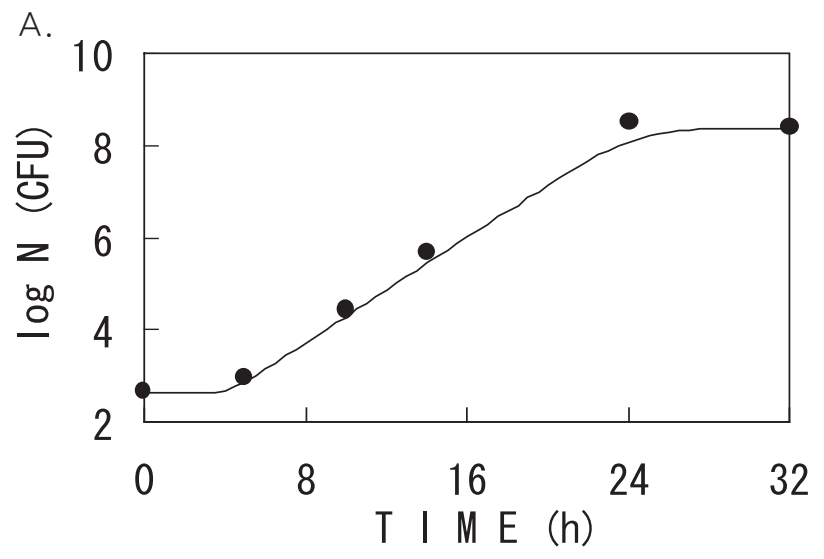

B.

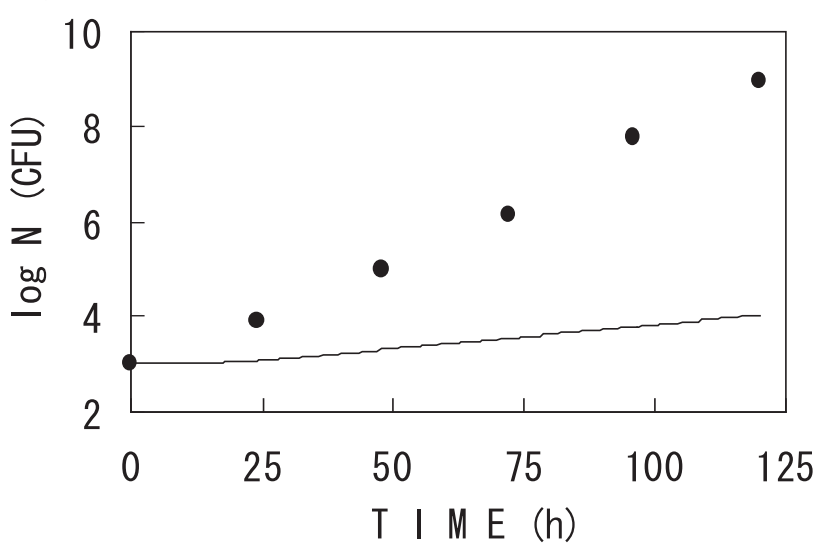

FIG. 2. Examples of growth predictions with parameters $k$ and $n$ estimated with the 3-order polynomial models. Growth conditions are A. $30^{\circ} \mathrm{C}, \mathrm{pH} 5.5$, and $6.5 \%$ salt and B. $30^{\circ} \mathrm{C}, \mathrm{pH} 6.0$, and $8.9 \%$ salt. Closed circles are experimental. Curves are described with the new logistic model and the 3-order polynomial models for parameters $k$ and $n$.

minimizing the MSE value between the observed and estimated bacterial populations in log units. The factors for $r$ and $n$ were estimated to be 1.0 and 1.3, respectively. Thus, the best estimates for $r$ and $n$ were obtained by multiplying the estimates with the 3-order equations by the corresponding factors. These improvements resulted in better predictions (Fig. 3). No poor predictions were made for any environmental conditions tested.

A predictive program for $V$. parahaemolyticus growth was then developed for use in the food industry. The program was built on our growth model (Eq. 1) and the polynomial equations for the growth parameters (Eq. 2). It was made using Microsoft Excel $2003^{\circledR}$.

When users want to predict $V$. parahaemolyticus growth, they first input the initial level of the organism, the environmental conditions including temperature, salt concentration, $\mathrm{pH}$, and the period of storage into 


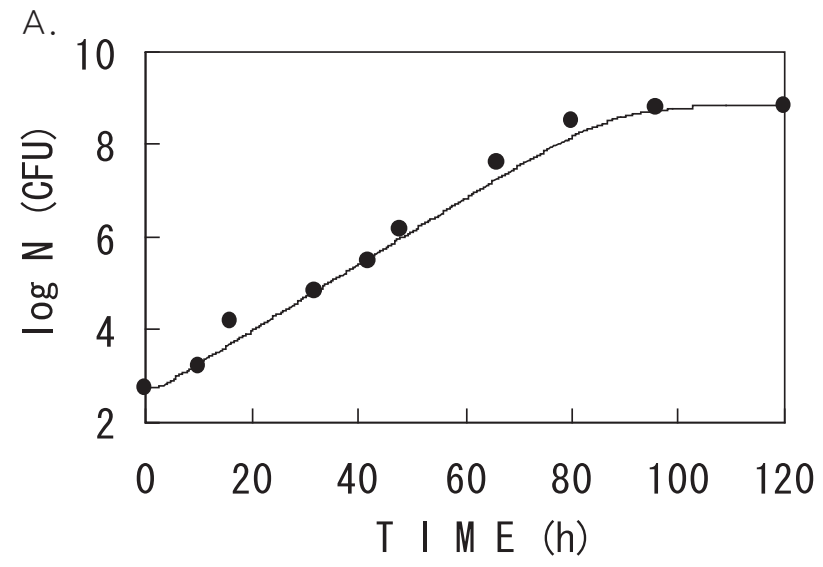

B.

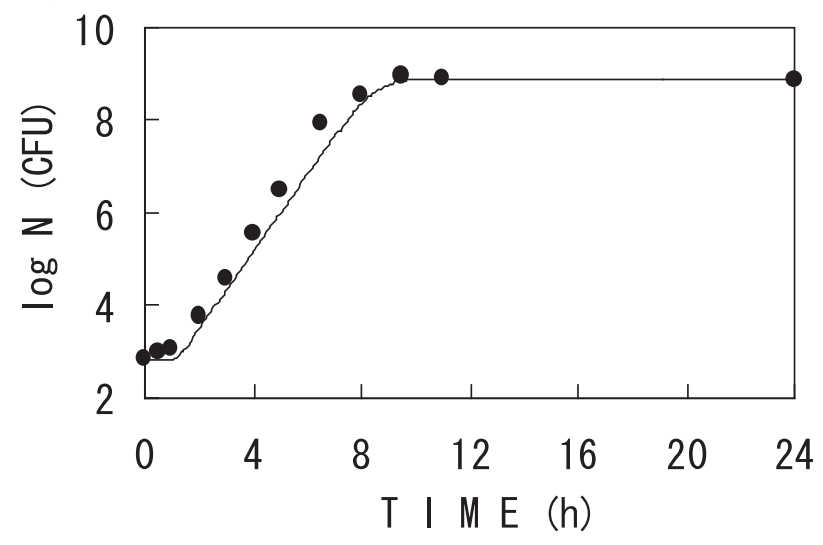

FIG. 3. Improved growth predictions. Growth conditions are A. $15^{\circ} \mathrm{C}, \mathrm{pH} 7.0$, and $6.5 \%$ salt and $\mathrm{B} .30^{\circ} \mathrm{C}, \mathrm{pH} 7.0$, and $4.5 \%$ salt. Closed circles are experimental. Curves are described with the new logistic model and the 3-order polynomial models for parameters $k$ and $n$.

the corresponding boxes (Fig. 4). The ranges of the environmental factors are shown near the boxes. Then, immediately after users push the prediction button, a predicted growth curve of the organism appears in the graph (Fig. 4). The graph can be printed out. Also, users can know the time for the bacteria to reach the level of concern and the level after the storage period of concern.

The data used for the predictive program development in this study were based on studies of bacteria cultured in broth, or well-conditioned media. This is because while there are a large number of seafood products to study, growth experiments for all such foods would be physically and economically impossible. There is a possibility that the program might predict greater or faster growth than the growth in real food. Therefore, users should know that the predicted growth shows the worst scenario from the viewpoint of food safety under the given conditions.

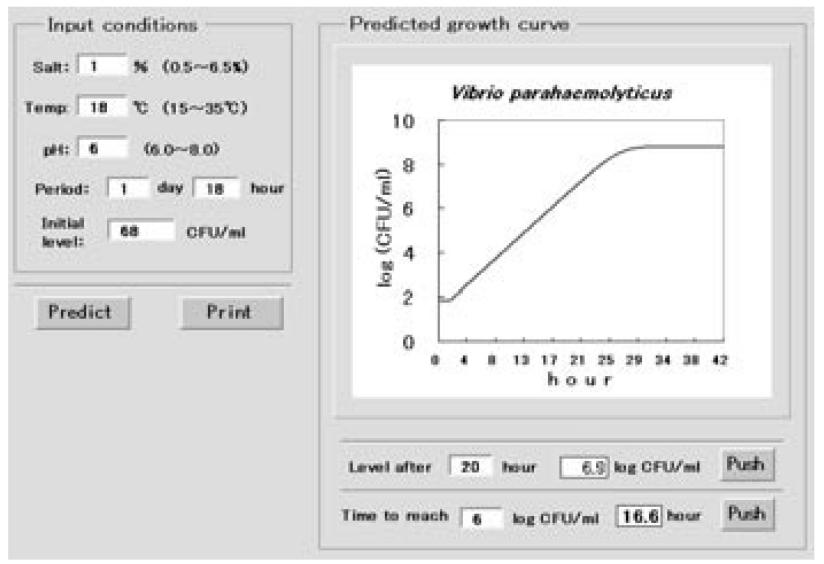

FIG. 4. V. parahaemoliticus predictive growth program. Immediately after the environmental conditions on salt concentration, temperature, $\mathrm{pH}$, the period of storage, and the initial bacterial level are input in the upper left boxes, a growth curve is generated in a graph. The bacterial level at the time of concern and the time to reach the bacterial level of concern are also predicted.

This program was developed using the growth data of one $V$. parahaemolyticus strain. Thus, it might be a prototype program for predicting bacterial growth. However, the program could be a predictive tool of $V$. parahaemolyticus growth in seafood products by including the growth data of more strains in a near future, because the structure of the prediction system was confirmed to be good in the present study.

\section{ACKNOWLEDGEMENTS}

The authors thank the Japan Food Industry Center for their financial support when we were conducting microbiological experiments and developing the prediction program.

\section{REFERENCES}

Baranyi, J. and Roberts, T. A. (1995) Mathematics of predictive food microbiology. Int. J. Food Microbiol., 26, 199218.

Buchanan, R. L., Whiting, R. C., and Damert, W.C. (1997) When is simple good enough: a comparison of the Gompertz, Baranyi, and three-phase linear models for fitting bacterial growth curves. Food Microbiol., 14, 313326.

Fujikawa, H., Kai, A., and Morozumi, S. (2003) A new logistic model for bacterial growth. J. Food Hyg. Soc. Japan, 44, 155-160.

Fujikawa, H., Kai, A., and Morozumi, S. (2004) A new logistic model for Escherichia coli at constant and dynamic temperatures. Food Microbiol., 21, 501-509.

Fujikawa, H. and Morozumi, S. (2005) Modeling Surface Growth of Escherichia coli on Agar Plates. Appl. Environ. Microbiol., 71, 7920-7926.

Fujikawa, H., Morozumi, S. (2007) Author's correction. Appl. Environ. Microbiol. 73, 2404. 
Fujikawa, H., Kano, Y. (2009) Development of a program to fit data to a new logistic model for microbial growth. Biocont. Sci. 14, 83-86.

Fujino, T., Okuno, Y., Nakada, D., Aoyama, A., Mukai, K., and Uebo, T. (1950) On the bacterial examination of Shirasu food poisoning. Med. J. Osaka Univ. 4, 299-304.

Gibson, A. M., Bratchell, N., and Roberts, T. A. (1987) The effect of sodium chloride and temperature on the rate and extent of growth of Clostridium botulinum type $A$ in pasteurized pork slurry. J. Appl. Bacteriol. 62, 479-490.

Kimura, B., Sekine, Y., Takahashi, H., Tanaka, Y., Obata, H., Kai, A., Morozumi, S., and Fujii, T. (2008) Multiple-locus variable-number of tandem-repeats analysis distin- guishes Vibrio parahaemolyticus pandemic O3:K6 strains. J. Microbiol. Meth. 72, 313-320.

Nair, G. B., Ramamurthy, T., Bhattacharya, S. K., Dutta, B., Takeda, Y., and Sack, D. A. (2007) Global dissemination of Vibrio parahaemolyticus serotype 03:K6 and its serovariants. Clin. Microbiol. Rev. 20, 39-48.

Okuda, J., Ishibashi, M., Hayakawa, E., Nishino, T., Takeda, Y., Mukhopadhyay, A. K., Garg, S., Bhattacharya, S. K., Nair, G. B., and Nishibuchi, M. (1997) Emergence of a unique 03:K6 clone of Vibrio parahaemolyticus in Calcutta, India, and isolation of strains from the same clonal group from Southeast Asian travelers arriving in Japan. J. Clin. Microbiol. 35, 3150-3155. 